\title{
The hypocretin/orexin system in sleep disorders: preclinical insights and clinical progress
}

\author{
This article was published in the following Dove Press journal: \\ Nature and Science of Sleep \\ 14 March 2016 \\ Number of times this article has been viewed
}

\author{
Matthew Chow \\ Michelle Cao \\ Department of Psychiatry and \\ Behavioral Sciences, Division of Sleep \\ Medicine, Stanford University School \\ of Medicine, Stanford, CA, USA
}

\begin{abstract}
Much of the understanding of the hypocretin/orexin (HCRT/OX) system in sleepwake regulation came from narcolepsy-cataplexy research. The neuropeptides hypocretin-1 and -2/orexin-A and -B (HCRT-1 and -2/OX-A and -B, respectively), as we know, are intimately involved in the regulation wakefulness. The HCRT/OX system regulates sleep-wake control through complex interactions between monoaminergic/cholinergic (wake-promoting) and gamma-aminobutyric acid-ergic (sleep-promoting) neuronal systems. Deficiency of HCRT/ OX results in loss of sleep-wake control or stability with consequent unstable transitions between wakefulness to nonrapid eye movement and rapid eye movement sleep. This manifests clinically as abnormal daytime sleepiness with sleep attacks and cataplexy. Research on the development of HCRT/OX agonists and antagonists for the treatment of sleep disorders has dramatically increased with the US Food and Drug Administration approval of the first-in-class dual HCRT/OX receptor antagonist for the treatment of insomnia. This review focuses on the origin, mechanisms of HCRT/OX receptors, clinical progress, and applications for the treatment of sleep disorders.
\end{abstract}

Keywords: hypocretin, orexin, narcolepsy, insomnia, orexin antagonist, orexin agonist

\section{Introduction}

Since de Lecea et $\mathrm{al}^{1}$ and Sakurai ${ }^{2}$ discovered hypocretin/orexin (HCRT/OX) in 1998, a body of literature investigating the distribution, molecular mechanism of action, physiologic role, disease activity, and potential for drug development of these large neuropeptides has emerged. A small but influential cluster of HCRT/OX neurons in the hypothalamus project to cholinergic and monoaminergic nuclei, which, in turn, project to the cerebral cortex promoting wakefulness. ${ }^{3}$ Disruption of this molecular mechanism may result in pathological sleep-wake states. Insomnia is a state of hyperarousal with difficulty falling asleep and maintaining sleep. Conversely, narcoleptics have unstable transitions from wakefulness to rapid eye movement (REM) sleep due to selective autoimmune destruction of HCRT/OX neurons. ${ }^{4}$ Dysfunction of the HCRT/OX system is also associated with alteration of energy homeostasis. In addition to unstable transitions between wakefulness and REM sleep, human narcoleptics have a higher incidence of metabolic syndrome with increased body mass index compared to controls. ${ }^{5}$ Studies evaluating HCRT/OX in terms of net energy balance are lacking in humans. Transgenic HCRT/OX-overexpressed mice demonstrate increased energy expenditure and reduced intake with prolonged exposure to high-fat diet. ${ }^{6}$ Interestingly, in the acute setting, HCRT/OX overexpression is associated with increased feeding behavior. There is even 
some literature to suggest that disruption of the HCRT/OX system may be therapeutic. HCRT/OX has been connected to the pathogenesis of Alzheimer's disease with decreased A $\beta$ plaque deposition seen in Amyloid Precursor Protein/Presenilin 1 transgenic mice, in which HCRT/OX has been knocked out. ${ }^{7}$ It is unclear whether this is mediated by disruption of the HCRT/OX system or a function of a parallel process associated with increased total sleep time. HCRT/OX has also been implicated in temperature regulation, sympathetic activation, analgesia, and mediating addiction. ${ }^{8}$

In light of these potential clinical targets, there has been ongoing academic and pharmaceutical interest in the development of selective HCRT/OX receptor agonists and antagonists. Given the high prevalence of insomnia, estimated to affect $10 \%-15 \%$ of the US adult population, ${ }^{9}$ industry efforts to develop an HCRT/OX receptor antagonist have been robust. In 2014, suvorexant became the first US Food and Drug Administration (FDA) approved HCRT/ OX receptor antagonist for the treatment of insomnia. ${ }^{10}$ In contrast, poor blood-brain barrier penetrance, complex interactions between HCRT/OX peptides and their associated G protein-coupled receptors (GPCRs), and the comparably smaller commercial market have slowed progress in the development of an HCRT/OX receptor agonist. In August 2015, Nagahara et $\mathrm{al}^{11}$ published their work in synthesizing the first HCRT/OX $\mathrm{R}$ agonist, compound 26, with good potency and selectivity.

According to American Academy of Sleep Medicine clinical guidelines, when the short-term use of a hypnotic agent is indicated for the treatment of primary insomnia, shortto intermediate-acting benzodiazepine receptor agonists (benzodiazepines and nonbenzodiazepine receptor agonists) are recommended as first-line therapy. Second-line therapy for primary insomnia includes sedating antidepressants, antiepileptics, and atypical antipsychotics, mainly as a secondary indication. While not recommended, antihistamines and other over-the-counter "sleep aids" are frequently used to treat primary insomnia. ${ }^{12}$ It remains to be seen whether suvorexant and other HCRT/OX receptor antagonists supplant these traditional hypnotics. Similarly, further animal studies are needed before HCRT/OX receptor agonists such as compound 26 are ready for human testing.

\section{The past and the future}

Despite their wide distribution of axonal projection and systemic effects, HCRT/OX-producing neurons are located in a small anatomical region of the posterolateral hypothalamus. This was suspected as early as 1930 by von Economo ${ }^{13}$ in the study of encephalitis lethargica patients after the H1N1 flu pandemic of 1918. It was not until the late 1990s that de Lecea et al $^{1}$ identified two peptide neurotransmitters derived from a propeptide precursor located in the synaptic vesicles of hypothalamic neurons. They termed these peptides hypocretin-1 and -2 (HCRT-1 and -2). Through an independent line of investigation, the Sakurai group identified the HCRT/OX receptors, locations of their messenger RNA expression in the central nervous system, and noticed increased feeding behavior in rodents that received intracerebroventricular (ICV) injection of HCRT-1/orexin-A (OX-A), ${ }^{2}$ thus naming them orexins for their presumed appetite stimulating property. Despite the difference in names, HCRTs and OXs are the same peptides. This experiment was the first therapeutic trial utilizing the HCRT/OX system, setting off a cascade of further investigation.

This small population of HCRT/OX-producing neurons exerts broad neuromodulatory effects, making it an interesting, yet challenging pharmacological target. HCRT/OX neurons project to neurochemically specific areas of the cerebral cortex, thalamus, hypothalamus, and brainstem with a variety of physiologic roles, in addition to wake promotion. A drug targeting such a system, whether it is an agonist or antagonist, faces the duality of possibly providing additional clinical benefits versus unanticipated side effects. While the vast majority of current research has revolved around the development of nonselective HCRT/OX receptor antagonists targeting insomnia, through the process of discovery, a number of promising compounds with different bioactivity have emerged.

\section{Receptor subtype location and activity}

There are two types of neuropeptides, HCRT-1/OX-A and HCRT-2/orexin-B (OX-B), acting on two types of receptors, HCRT/OX R and HCRT/OX R. The HCRT/OX 2 R nonselectively binds both HCRT-1/OX-A and HCRT-2/OX-B. The HCRT/OX $\mathrm{R}$ is selective for HCRT-1/OX-A. ${ }^{14}$ In addition to this differential binding affinity, the HCRT/OX and its associated receptors are differentially distributed in the brain. HCRT/OX $\mathrm{R}$ is found densely in cholinergic neurons in the pedunculopontine and lateral dorsal tegmental nuclei, and is the only HCRT/OX receptor subtype in adrenergic neurons of the locus coeruleus. HCRT/OX $\mathrm{R}$ and HCRT/OX $\mathrm{R}$ are both found in serotonergic dorsal raphe neurons and dopaminergic ventral tegmental area neurons. HCRT/OX 2 is the only HCRT/OX receptor subtype in the histaminergic tuberomammillary nucleus. ${ }^{15}$ 
The preferential receptor subtype expression and ligandbinding affinity in different cholinergic and monoaminergic pathways suggests HCRT/OX R and HCRT/OX $\mathrm{O}_{2}$ have different physiologic roles. This has been demonstrated in both rodent and canine models. In addition to promoting feeding behavior, ICV administration of HCRT-1/OX-A in wild-type mice increases wakefulness and reduces both REM and nonrapid eye movement (NREM) sleep. ${ }^{16}$ In contrast, ICV HCRT-1/OX-A given to HCRT/OX ${ }_{1}$ R and HCRT/OX 2 R knockout mice fails to produce the same effect. These knockout mice have reduced wakefulness and NREM sleep, with the HCRT/OX 2 R knockout mice being the most affected. REM sleep suppression was slightly and similarly attenuated in both knockout mice subtypes. This suggests both receptors are important in the maintenance of wakefulness and NREM sleep, with HCRT/OX ${ }_{2} \mathrm{R}$ playing the dominant role. While HCRT/OX 2 R and HCRT/OX knockout mice both have attenuated wakefulness, $\mathrm{HCRT} / \mathrm{OX}_{2} \mathrm{R}$ knockout mice are less severely affected by cataplexy while HCRT/OX knockout mice have an increased tendency for unstable transitions from wakefulness to REM sleep. ${ }^{17}$ Similarly, observations in familial canine narcolepsy with $\mathrm{HCRT} / \mathrm{OX}_{2} \mathrm{R}$ mutation have shown normal cerebrospinal fluid (CSF) levels of HCRT-1/OX-A and HCRT-2/OX-B with intact HCRT/OX ${ }_{1}$, suggesting a more prominent role of $\mathrm{HCRT} / \mathrm{OX}_{2} \mathrm{R}$ in the narcoleptic phenotype in dogs. ${ }^{18}$

\section{Development of the first dual OX receptor antagonist}

Preclinical studies of suvorexant administered to rats, dogs, and Rhesus monkeys showed a temporary and dosedependent sleep-promoting effect, spurring further clinical studies in humans. ${ }^{19}$ Several randomized, double-blind, placebo-controlled trials have been conducted evaluating the efficacy, safety, and tolerability of suvorexant in the treatment of insomnia. In one study, 249 patients were randomized to one of four different doses $(10,20,40$, and $80 \mathrm{mg})$ to be taken over two 4 -week periods. They were assessed via polysomnograms on days 1 and 28 for both 4 -week periods. The results showed a dose-dependent improvement in total sleep time, sleep latency, and wake time after sleep onset. The most commonly reported adverse effect was somnolence, with only one person reporting next day somnolence on the $80 \mathrm{mg}$ dose. ${ }^{20} \mathrm{~A}$ similar crossover study was conducted in young healthy men who underwent polysomnograms for five 2-night periods in which they were given doses of 10, 50, $100 \mathrm{mg}$, or placebo on the second night. The fifth night was evaluated only for pharmacokinetic assessment. Sleep periods were separated by a 96-hour washout period. Similar to the previous study; sleep latency, total sleep time, and wake time after sleep onset improved. Next day residual sleepiness was significant only at the $100 \mathrm{mg}$ dose. ${ }^{21}$ Another study looked at the long-term efficacy, safety, and tolerability of suvorexant. Patients were studied for a 1-year period with a 2-month discontinuation phase to assess for withdrawal effects. Patients $<65$ years old received a $40 \mathrm{mg}$ dose and patients older than 65 years received a $30 \mathrm{mg}$ dose. Subjective total sleep time and sleep latency was assessed at 1 month. The medication was well tolerated without significant withdrawal effects. At 1 month, patients taking suvorexant reported improved sleep onset and total sleep time compared to controls. ${ }^{22}$ Lower $20 \mathrm{mg}(<65$ years old $) / 15 \mathrm{mg}(>65$ years old $)$ doses were also investigated. While the $40 \mathrm{mg}$ ( $<65$ years old) $/ 30 \mathrm{mg}$ ( $>65$ years old) doses were modestly more effective in terms of subjective and objective sleep onset and maintenance, the lower dosing scheme was adequately effective with less daytime somnolence, although the incidence of this was low overall. ${ }^{23}$

Suvorexant is a first-in-class FDA-approved dual HCRT/OX receptor antagonist. Subjective and objective measures of its hypnotic effect in humans were demonstrated in several clinical trials. Review of the Herring et $\mathrm{al}^{20}$ data shows a strong first night effect in a dose-dependent fashion compared to placebo in terms of total sleep time, wake after sleep onset, and sleep latency. Less effect was seen when comparing the $40 \mathrm{mg}$ to the $80 \mathrm{mg}$ dose. The greatest total sleep time change occurred at the $20 \mathrm{mg}$ dose at the end of the 4 -week period. Based on the Sun et $\mathrm{a}^{21}$ data, in healthy young men of age 18-45 years, next day somnolence was seen at a rate of $4.5 \%, 4.8 \%$, and $20.5 \%$, for the 10,50 , and $100 \mathrm{mg}$ dosing, respectively. The most common side effects were somnolence, fatigue, and headache. Rarely, it caused abnormal dreams, hallucinations, sleep paralysis, and motor incoordination. In 1-year follow-up study of adult patients with a Diagnostic and Statistical Manual of Mental Disorders$I V$-Text Revision diagnosis of primary insomnia and taking suvorexant $40 \mathrm{mg}$ ( $<65$ years old) or $30 \mathrm{mg}$ ( $>65$ years old) compared to placebo, the patients taking suvorexant most commonly reported increased somnolence $(13.2 \%$ vs $2.7 \%$ ), fatigue ( $6.5 \%$ vs $1.9 \%$ ), or dry mouth ( $5 \%$ vs $1.6 \%$ ) compared to placebo. ${ }^{22}$ Due to the concern for possible next day somnolence as well as the desire to find the minimally effective dose, the FDA recommended a dosing range of 5-20 $\mathrm{mg}$. There is some suggestion that while similarly "likeable" among recreational drug abusers at high doses when compared with zolpidem, suvorexant had reduced abuse potential. ${ }^{10}$ 
Approval for the clinical use of suvorexant in humans is significant for several reasons:

- It gives patients an alternative to traditional sedative/ hypnotic agents that tend to have more cognitive side effects and withdrawal symptoms.

- The commercial applications of HCRT/OX agonists and antagonists will stimulate further research development.

- It affords a larger population from which to collect additional data on the medication's effects. However, cost will likely be a limiting factor in suvorexant's widespread use as existing sleeping aids have become fairly inexpensive.

\section{Clinical investigation of dual (type $I$ and 2) and selective type 2 HCRT/OX receptor antagonists as hypnotics}

The dual and selective HCRT/OX 2 R antagonists have been targeted for clinical development primarily for their sedating properties. Suvorexant (MK-4305) may have been the first dual HCRT/OX receptor antagonist approved by the FDA for the treatment of insomnia; however, it was not the first to be clinically investigated in humans. Two selective $\mathrm{HCRT} / \mathrm{OX}_{2} \mathrm{R}$ antagonists have been tested in humans, MK-1064 and JNJ-42847922. Both agents demonstrated a dose-dependent reduction in alertness/increased sleepiness. On polysomnogram, MK-1064 increased sleep efficiency and reduced sleep latency without affecting NREM/REM balance. ${ }^{24}$ Other than somnolence, headache and dizziness were the most commonly reported side effects of JNJ-42847922. Interestingly, one subject who received $80 \mathrm{mg}$ of JNJ-42847922 experienced a single brief episode of sleep paralysis following administration. ${ }^{25}$ The pharmaceutical company Actelion initially introduced almorexant (ACT-078573), a nonselective HCRT/OX receptor antagonist. In Phase I, the medication proved effective, showing a threshold dose-dependent increase in sleepiness. It was also well tolerated with doses up to 1,000 mg failing to induce narcolepsy or cataplexy type symptoms. Doses over $200 \mathrm{mg}$ saw an increase in somnolence, dizziness, inattention, and fatigue. In Phase II, doses of 200-400 mg were administered. Polysomnographic data showed increased sleep efficiency and total sleep time with shorter sleep latency and wake time after sleep onset. Given the promising Phase II results, almorexant underwent Phase III clinical testing. In Phase III (RESTORA1), the trial was discontinued due to transient liver function test abnormalities. $^{26}$
Similar polysomnographic data were obtained in Phase II clinical trials of another dual HCRT/OX receptor antagonist, SB-649868. ${ }^{27}$ This compound showed similar effect to benzodiazepine receptor agonists and was well tolerated. However, it was a potent cytochrome P450 3A4 inhibitor and further clinical study was discontinued. Filorexant (MK-6096) is a dual HCRT/OX receptor antagonist that has been investigated for a number of conditions including insomnia, neuropathic pain, adjunctive treatment for depression, and migraine prophylaxis. The only published human clinical data on the medication was a randomized control trial of its use in migraine prophylaxis. It failed to demonstrate efficacy in migraine prophylaxis, but not unexpectedly, patients reported increased somnolence (13\%) compared to control (4\%). ${ }^{28}$

\section{Other nonhypnotic effects of selective type I and type 2 HCRT/OX receptor antagonists}

While dual and selective HCRT/OX ${ }_{2} \mathrm{R}$ antagonists have mainly been investigated for their hypnotic effect, some interesting work has been done on HCRT/OX receptor subtypespecific drugs in animal models. The first such molecule was a selective HCRT/OX $\mathrm{R}$ antagonist discovered by researchers from the pharmaceutical company GlaxoSmithKline and named as SB-334867. It was found to reduce HCRT-1/OX-Ainduced grooming behavior in rats without significant sedation. ${ }^{29}$ The first HCRT/OX ${ }_{2} \mathrm{R}$ antagonist was JNJ-1037049. This molecule was found to have hypnotic effects and reduced reward-seeking behavior for alcohol in rats. ${ }^{30}$ ACT-335827 is a selective $\mathrm{HCRT} / \mathrm{OX}_{1} \mathrm{R}$ antagonist with anxiolytic properties in rats. ${ }^{31}$ Early animal studies on selective HCRT/OX receptor antagonists are important on several fronts:

- The HCRT/OX neural pathways exert physiologic effects beyond simply the regulation of sleep-wake.

- These physiologic effects are influenced by regional receptor subtype density.

- They offer promising clinical indications for behavioral modification via a novel mechanism that has yet to be explored.

\section{Clinical indications for an HCRT/OX receptor agonist}

HCRT/OX deficiency (narcolepsy) manifests classically with the tetrad of cataplexy, excessive daytime sleepiness, sleep paralysis, and hypnagogic/hypnopompic hallucinations. ${ }^{32}$ Automatic behaviors and sleep fragmentation are also common clinical features. Other sleep disorders such as obstructive sleep apnea, restless leg syndrome, and 
REM sleep behavior disorder are more common in narcoleptics, primarily those with cataplexy (type 1), relative to the general population. ${ }^{33}$ They also have more medical (hypercholesterolemia, gastrointestinal disease, heart disease, upper respiratory tract disease, and hypertension) and psychiatric (anxiety and depression) comorbidities ${ }^{34}$ with higher utilization of health services. ${ }^{35}$ CSF HCRT-1/OX-A is undetectable in up to $95 \%$ of patients with type 1 narcolepsy ${ }^{36}$ with absence of HCRT/OX and its precursor peptide at autopsy. Selective destruction of the HCRT/OX neurons with preservation of proximate structures suggests a highly specific autoimmune pathophysiology. The multiple sleep latency test, human leukocyte antigen testing, and CSF sampling have increased diagnostic certainty; however, current guideline therapy for narcolepsy remains limited. Stimulants, the sedative/hypnotic medication sodium oxybate, activating antidepressants, and scheduled naps are the mainstay of therapy. The side effect profile and abuse potential of these medications make them less than ideal. There is an unmet need for a selective HCRT/OX receptor agonist with high receptor affinity and central nervous system penetrance. Of note, it is conceivable that patients with hypersomnia unrelated to narcolepsy may benefit from an HCRT/OX agonist as well; however, this is often secondary to an undiagnosed comorbid sleep disorder, medication, or other medical condition for which more established treatments exist. While ICV and intravenous administration of HCRT/OX have shown to reverse the clinical effects of narcolepsy in animal models, ${ }^{37}$ this is clinically not practical in humans.

\section{A selective $O X_{2} R$ agonist}

Recently, Nagahara et $\mathrm{al}^{11}$ described the screening and synthesis of a novel compound $26\left(\mathrm{EC}_{50}=0.023 \mu \mathrm{M}, \mathrm{E}_{\max }=98 \%\right.$ in HCRT/OX 2 R). It is hypothesized that compound 26 acts as a "molecular switch", engaging GPCRs and causing a conformational change. It does this by mediating the relative position of transmembrane helices 5 and 6 in relationship to the rest of the transmembrane bundle. This then activates an intracellular signaling cascade. ${ }^{38}$ While suvorexant inhibits the inward movement of these helices, exerting an antagonistic effect on the $\mathrm{HCRT} / \mathrm{OX}_{2} \mathrm{R}$, compound 26 facilitates this movement, acting as a potent and selective agonist. Hopefully, the discovery of compound 26 will spur the development of preclinical models and derivative molecules which activate HCRT/OX GPCRs. High throughput screening assays have identified a large number of candidate compounds. Small chemical structural variations can change the binding selectivity of these compounds for HCRT/OX receptor subtypes. This has led to the identification of a number of promising molecules for clinical development.

\section{Discussion}

HCRT/OX neurons activate wake-promoting regions of the brain. The distribution of HCRT/OX subtypes helps to determine its physiologic function. When things go awry, this can result in disorders of arousal. Insomnia and narcolepsy are prime examples of this, but on opposite ends of the clinical spectrum. This has led to the development of a number of dual and selective HCRT/OX receptor antagonists as well as a single HCRT/OX 2 R agonist. Dual and HCRT/OX 2 R antagonists have shown the most clinical promise in the treatment of insomnia. The FDA recently approved suvorexant, a dual HCRT/OX receptor antagonist. HCRT/OX 2 R antagonists are currently in clinical development. While the majority of research in the field has focused on the sleep-wake system, preclinical experiments in animals have shown that HCRT-1/OX-A increases appetite and energy expenditure, an $\mathrm{HCRT} / \mathrm{OX}_{2} \mathrm{R}$ antagonist reduces reward-seeking behavior, and an HCRT/OX $\mathrm{R}$ antagonist has anxiolytic properties. Further study is needed to elucidate the physiologic effects of the HCRT/OX system. We are now only beginning to develop therapeutic agents specifically targeting this system.

\section{Disclosure}

The authors report no conflicts of interest in this work.

\section{References}

1. de Lecea L, KilduffTS, Peyron C, et al. The hypocretins: hypothalamusspecific peptides with neuroexcitatory activity. Proc Natl Acad Sci USA. 1998;95(1):322-327.

2. Sakurai T. Orexins and orexin receptors: implication in feeding behavior. Regul Pept. 1999;85(1):25-30.

3. Hungs M, Mignot E. Hypocretin/orexin, sleep and narcolepsy. Bioessays. 2001;23(5):397-408.

4. Mahlios J, De la Harrán-Arita AK, Mignot E. The autoimmune basis of narcolepsy. Curr Opin Neurobiol. 2013;23(5):767-773.

5. Nishino S, Ripley B, Overeem S, et al. Low cerebrospinal fluid hypocretin (orexin) and altered energy homeostasis in human narcolepsy. Ann Neurol. 2001;50(3):381-388.

6. Funato H, Tsai AL, Willie JT, et al. Enhanced orexin receptor-2 signaling prevents diet-induced obesity and improves leptin sensitivity. Cell Metab. 2009;9(1):64-76.

7. Roh JH, Jiang H, Finn MB, et al. Potential role of orexin and sleep modulation in the pathogenesis of Alzheimer's disease. J Exp Med. 2014;211(13):2487-2496.

8. Kukkonen JP. Recent progress in orexin/hypocretin physiology and pharmacology. Biomol Concepts. 2012;3(5):447-463.

9. WB\&A Market Research. 2005 Sleep in America poll. Washington, DC: National Sleep Foundation; 2005:1-55. Available from: https:// sleepfoundation.org/sites/default/files/2005_summary_of_findings. pdf.

10. Patel KV, Aspesi AV, Evoy KE. Suvorexant: a dual orexin receptor antagonist for the treatment of sleep onset and sleep maintenance insomnia. Ann Pharmacother. 2015;49(4):477-483. 
11. Nagahara T, Saitoh T, Kutsumura N, et al. Design and synthesis of non-peptide, selective orexin receptor 2 agonists. $J$ Med Chem. 2015;58(20):7931-7937.

12. Schutte-Rodin S, Broch L, Buysse D, Dorsey C, Sateia M. Clinical guideline for the evaluation and management of chronic insomnia in adults. J Clin Sleep Med. 2008;4(5):487-504.

13. von Economo C. Sleep as a problem of localization. J Nerv Ment Dis. 1930;71(3):1-5.

14. Sakurai T, Amemiya A, Ishii M, et al. Orexins and orexins receptors: a family of hypothalamic neuropeptides and $\mathrm{G}$ protein-coupled receptors that regulate feeding behavior. Cell. 1998;92:573-585.

15. Nunez A, Rodrigo-Angulo ML, De Andres I, Garzon M. Hypocretin/ orexin neuropeptides: participation in the control of sleep-wakefulness cycle and energy homeostasis. Curr Neuropharmacol. 2009;7(1):50-59.

16. Mieda M, Hasegawa E, Kisanuki YY, Sinton CM, Yanagisawa M, Sakurai T. Differential roles of orexin receptor-1 and -2 in the regulation of non-REM and REM sleep. J Neurosci. 2011;31(17):6518-6526.

17. Willie JT, Chemelli RM, Sinton CM, et al. Distinct narcolepsy syndromes in orexin receptor-2 and orexin null mice: molecular genetic dissection of non-REM and REM sleep regulatory processes. Neuron. 2003;38(5):715-730.

18. Ripley B, Fujiki N, Okura M, Mignot E, Nishino S. Hypocretin levels in sporadic and familial cases of canine narcolepsy. Neurobiol Dis. 2001;8(3):525-534.

19. Winrow C, Gotter A, Cox C, et al. Promotion of sleep by suvorexanta novel dual orexin receptor antagonist. $J$ Neurogenet. 2011;25(1-2): $52-61$.

20. Herring JW, Snyder E, Budd K, et al. Orexin receptor antagonism for treatment of insomnia: a randomized clinical trial of suvorexant. Neurology. 2012;79:2265-2274.

21. Sun H, Kennedy WP, Darren W, et al. Effects of suvorexant, an orexin receptor antagonist, on sleep parameters as measured by polysomnography in healthy men. Sleep. 2013;36:259-267.

22. Michelson D, Snyder E, Paradis E, et al. Safety and efficacy of suvorexant during 1-year treatment of insomnia with subsequent abrupt treatment discontinuation: a phase 3 randomised, double-blind, placebo-controlled trial. Lancet Neurol. 2014;13:461-471.

23. Herring J, Connor K, Snyder E, et al. Suvorexant in patients with insomnia: results from two 3-month randomized controlled clinical trials. Biol Psychiatry. 2016;79(2):136-148.

24. Coleman PJ, Cox CD, Breslin MJ, et al. Discovery and characterization of $\mathrm{OX}_{2} \mathrm{R} / \mathrm{OX}_{1} \mathrm{R}$ and $\mathrm{OX}_{2} \mathrm{R}$ antagonists for the treatment of sleep disorders. ACS Natl Mtg. 2014.
25. Bonaventure P, Shelton J, Yun S, et al. Characterization of JNJ-42847922, a selective orexin-2 receptor antagonist, as a clinical candidate for the treatment of insomnia. J Pharmacol Exp Ther. 2015;354(3):471-482.

26. Roecker AJ, Cox CD, Coleman PJ. Orexin receptor antagonists: new therapeutic agents for the treatment of insomnia. J Med Chem. 2016;59(2):504-530.

27. Bettica P, Squassante L, Zamuner S, Nucci G, Danker-Hopfe H, Ratti E. The orexin antagonist SB-649868 promotes and maintains sleep in men with primary insomnia. Sleep. 2012;35(8):1097-1104.

28. Chabi A, Zhang Y, Jackson S, et al. Randomized controlled trial of the orexin receptor antagonist filorexant for migraine prophylaxis. Cephalagia. 2015;35(5):379-388.

29. Duxon MS, Stretton J, Starr K, et al. Evidence that orexin-A-evoked grooming in the rat is mediated by orexin-1 (OX1) receptors, with downstream 5-HT2C receptor involvement. Psychopharmacology (Berl). 2001;153(2):203-209.

30. Shoblock JR, Welty N, Aluisio L, et al. Selective blockade of the orexin-2 receptor attenuates ethanol self-administration, place preference, and reinstatement. Psychopharmacology (Berl). 2011;215(1):191-203.

31. Steiner MA, Gatfield J, Brisbare-Roch C, et al. Discovery and characterization of ACT-335827, an orally available, brain penetrant orexin receptor type 1selective antagonist. ChemMedChem. 2013;8(6): 898-903.

32. Bourgin P, Zeitzer JM, Mignot E. CSF hypocretin-1 assessment in sleep and neurological disorders. Lancet Neurol. 2008;7(7):649-662.

33. Nevsimalova S, Pisko J, Buskova J, et al. Narcolepsy: clinical differences and association with other sleep disorders in different age groups. J Neurol. 2013;260(3):767-775.

34. Ohayon MM. Narcolepsy is complicated by high medical and psychiatric comorbidities: a comparison with the general population. Sleep Med. 2013;14(6):488-492.

35. Black J, Reaven NL, Funk SE, et al. The Burden of Narcolepsy Disease (BOND) study: health-care utilization and cost findings. Sleep Med. 2014;15(5):522-529.

36. Nishino S, Ripley B, Overeem S, Lammers GJ, Mignot E. Hypocretin (orexin) deficiency in human narcolepsy. Lancet. 2000;355:39-40.

37. Fujiki N, Yoshida Y, Ripley B, Mignot E, Nishino S. Effects of IV and ICV hypocretin-1 (orexin A) in hypocretin receptor-2 gene mutated narcoleptic dogs and IV hypocretin-1 replacement therapy in a hypocretinligand-deficient narcoleptic dog. Sleep. 2003;26:953-959.

38. Heifetz A, Bodkin MJ, Biggin PC. Discovery of the first selective, nonpeptidic orexin 2 receptor agonists. $J$ Med Chem. 2015;58(20): 7928-7930.
Nature and Science of Sleep

\section{Publish your work in this journal}

Nature and Science of Sleep is an international, peer-reviewed, open access journal covering all aspects of sleep science and sleep medicine, including the neurophysiology and functions of sleep, the genetics of sleep, sleep and society, biological rhythms, dreaming, sleep disorders and therapy, and strategies to optimize healthy sleep. The journal welcomes

\section{Dovepress}

original research, clinical \& epidemiological studies, reviews \& evaluations, case reports and extended reports. The manuscript management system is completely online and includes a very quick and fair peerreview system, which is all easy to use. Visit http://www.dovepress.com/ testimonials.php to read real quotes from published authors. 